\title{
Tunable holographic components in WDM optical networks
}

\author{
Alfredo Martín Minguez • Paloma R. Horche
}

\begin{abstract}
This paper describes the applications of a multipurpose holographic device in optical networks with Coarse and Dense Wavelength Division Multiplexing (CWDM/ DWDM) technologies. In its basic structure, it can operate as a tunable wavelength filter, wavelength multiplexer or $\lambda$ router. By using a more complex structure, the device works as OADM (Optical Add Drop Multiplexer) or OS (Optical Switch). Some simulations of the basic devices, from the optical transmission point of view, are made to match the transmission parameters for the application in optical networks. Performance parameters of the device, like switching time, losses, cross-talk or polarization insensitivity are analyzed and compared with other multiplexing or switching technologies. To complete the review of these components, a study of computer generated holograms (CGH) design is carried out. The results are used in the design of holographic devices to perform different applications: in Metro networks, where a design of a holographic device with wavelength conversion and routing is analyzed, or, in Access Networks like a tunable filter or demultiplexer in Fiber to the Home/Business (FTTH/FTTB) topologies.
\end{abstract}

Keywords WDM optical networks - Holographic device - Optical filter - Optical router . Optical switch - Semiconductor optical amplifier - SOA - Access network + Metro network

\section{Introduction}

Wavelength Division Multiplexing (WDM) technologies are being widely deployed internationally, since it is recognized that they can satisfy the traffic demand for high-capacity networking. The last few years have also marked the introduction of DWDM (Dense

\footnotetext{
A. Martín Minguez $(\bowtie) \cdot$ P. R. Hotche Dpto. de Tecnología Fotónica (ETSI Telecomunicación), Univetsidad Politécnica de Madrid. C.udad Universitatia s/n, 28040 Madrid, Spain e-mail: alfredo.minguez@tfo.upm.es

P. R. Horche

e-mail: photche 0 tfo.upm.es
} 
Wavelength Division Multiplexing) technology in metropolitan and access networks due to the increased demand for delivering more bandwidth to the subscriber, created by the need of enhanced services.

For Metro, and mainly for Access networks applications, an increment in capacity may be achieved with a cost-effective multiplexing technology without the need for the high channel counts and closely spaced wavelengths typically used in long haul networks. A channel space of 5-30 nm can be used, relaxing the processing tolerances and potentially lowering the cost of components. CWDM (Coarse Wavelength Division Multiplexing) technology reaches those requirements and it has been proposed for these applications. It is in this context where this multipurpose holographic device has different use in Metro or access optical networks (Martín Minguez and Horche 2007a).

In this paper some applications of the holographic WDM multifunction device in METRO networks are described, such as the utilization of OADMs in an optical path protection/reconfiguration between nodes $(1+1$ configuration) and the use of optical switches to interconnect nodes of the METRO-Access network with a METRO-Core or long haul networks in a reconfigurable topology.

Other applications in Access networks are commented: the use of OADMs in the optical path protection between the OLT (Optical Line Termination) at the CO (Central Office) and the RN (Remote Node) in a FTTO-PON (Fiber to the Office Passive-Optical Network) or; in some specific cases, the utilization as tunable holographic filters in a FTTO application, at the Business ONT (BONT), to select the assigned optical wavelength in agreement with the services provided to the customer.

This paper is a summary of a most exhaustive work (Martín Minguez 2007). The emphasis here is, on one hand, in the main design guidelines of the holographic device and its holograms, and, on the other hand, in its network applications, like the design of a holographic device with wavelength conversion and routing, with losses compensated by the gain of a SOA, which simulation in a Metro network has been done.

The structure of the paper is the following: Section 2 deals with the design of the holographic device which main component is a ferroelectric liquid crystal (FLC) spatial light modulator (SLM) where dynamic holograms are implemented in real time. In Section 3 are described some guidelines for the design of holograms by computer to be used in SLMs. Section 4 describes some applications (filters, demultiplexers, routers) for the basic structure and the corresponding simulations. Section 5 shows the composed structures applications such as OADMs or switch matrixes. In Section 6, some considerations about performance parameters for these components and the impact in its network applications are done. Section 7 addresses typical applications of the device, like filter, router, OADM or switch, in Metro and Access networks. Finally, in Section 8 a comparison of different technologies for OADMs is done. Some comments for an optimized holographic Reconfigurable OADM (ROADM) design are also included.

\section{Holographic WDM device design}

The operating principle of the dynamic holographic device is based in the wavelength dispersion produced in a diffraction grating component. When a polychromatic light reaches a diffraction grating, there is an angular dispersion (diffraction) according to the wavelength of the incident light; the equation (1) takes the relationship between the diffraction angle and the wavelength $\lambda$ into account (Kashnow and Bigelow 1973): 


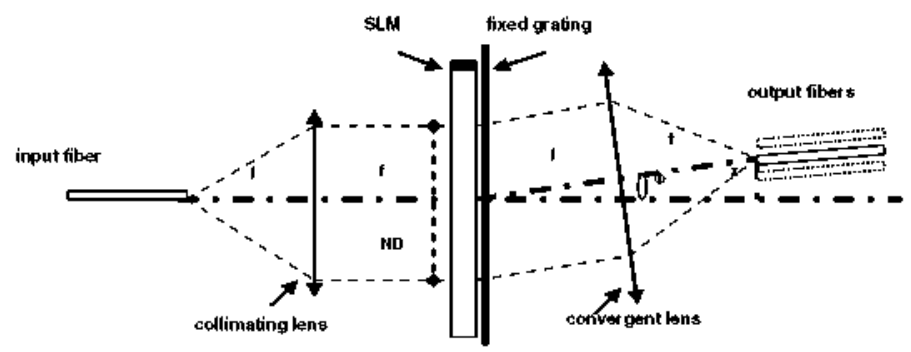

(a) Tunable holographic device

\begin{tabular}{|c|c|c|}
\hline D & $x$ & Application \\
\hline fix & fix & Holographic bandpass filter \\
\hline variable & fix & $\begin{array}{c}\text { Turnable hologa aplic } \\
\text { bandpass filter }\end{array}$ \\
\hline fix & variable & Demultiplexer $1 \times \mathrm{m}$ \\
\hline variable & variable & $\lambda$ router $1 \times \mathbf{m}$ \\
\hline
\end{tabular}

(b) Tunable holographic device applications

Fig. 1 Tunable holographic device: a physical structure; b WDM applications

$$
\sin \beta=\frac{m \lambda}{d}
$$

By considering the incident light perpendicular to the grating, $\beta$ is the diffracted light angle, $m$ is the diffraction order and $d$ the grating spatial period. Usually, $m=+1$ is considered because it is the first diffraction order with the maximum intensity; in (1) $d>\lambda$ has to be reached.

According to (1), if there is a change in the spatial period $d$ or in the incident light wavelength $\lambda$, the diffraction angle $\beta$ also changes. Consequently, we can implement a wavelength tunable optical filter, demultiplexer or $\lambda$ router by using the characteristics of this structure.

The former structure does not have possibility, in a practical way, for changing the spatial period or the wavelength. One of the devices that allows these variations is a Spatial Light Modulator (SLM), where every ferroelectric liquid crystal (FLC) pixel can be electro-optically configured to provide a phase modulation to the incident light. On this way, managing a hologram on the SLM, where its spatial period can be modified, we obtain a programmable diffraction grating (Martín Minguez and Horche 2005).

For CWDM/DWDM systems applications where the different channels are separated $20 / 0.8 \mathrm{~nm}$ with a central wavelength around $1.441 / 1.551 \mu \mathrm{m}$, the current commercial SLMs do not have enough resolution (the pixel size has to be in the order of the wavelength range); therefore, the solution is to use a fixed diffraction grating, with a low spatial period together with the SLM, giving a high resolution filter.

With this structure, in Fig. 1a, Parker et al. $(1998,1997)$, the SLM produces small changes in the light diffraction angle and the fixed grating selects the required wavelengths. The architecture uses a phase SLM and a fix grating, both of them transmissive. The name "lineal $4 f$ ", results from the distance $4 f$ between the input and the output of the filter, where $f$ is the focal distance of the lens.

The output fiber(s) are placed at the lens focal plane where the Fourier transform of the hologram, array of light spots for a black and white bars hologram, is located. The SLM_FLC and fixed grating are illuminated by a collimated light coming from a single mode optical 
fiber, through a lens; a second lens couples the first order diffracted light in an output optical fiber(s) like a spatial light filter.

There are two parameters that impact strongly in the size of the filter: the lens focal distance, $f$, and the product $N D$, where $D$ is the size of the pixel and $N$ is the number of pixels in one dimension of the SLM.

The relationship between the hologram spatial period, $H$, and $N D$ is:

$$
H=\frac{N D}{n} \quad 0<n<\frac{N}{2} n, \text { integer }
$$

where $n$ depends on the type of hologram, black/white bars for this application.

In equation (3), Parker et al. (1998), according to the value of $n$ we can select different $\lambda^{\prime} s$ at the end of the filter in the output fiber:

$$
\lambda \approx \frac{x}{f} \cdot \frac{1}{\left(\frac{n}{N D}+\frac{1}{d}\right)}
$$

where $x$ is the distance of the output optical fiber to the optical axis and $d$ is the spatial period of the fixed grating.

In Fig. Ib we show a table where four different applications: holographic band pass filter, tunable holographic band pass filter, demultiplexer $1 \times m$ and $\lambda$ router $1 \times m$, are possible for this multipurpose device. All of them use the same holographic structure, and the type of application depends on the fixed or variable values for $n$ and $x$ (Martín Minguez and Horche 2005). This is one of the novel features developed for the designed device.

In Fig. 2, the basic holographic device applications, tunable filters, demultiplexers and routers, are illustrated. The design roules for holographic demultiplexers and routers take equations (4) and (5) into account, Martín Minguez and Horche (2005), both of them coming from (3):

$$
\begin{aligned}
x & \approx \lambda f\left(\frac{n}{N D}+\frac{1}{d}\right) \Rightarrow \\
\Delta x & \approx \Delta \lambda f\left(\frac{n}{N D}+\frac{1}{d}\right) \\
n & \approx\left(\frac{x}{\lambda f}-\frac{1}{d}\right) N D \Rightarrow \Delta n \approx\left(\frac{\Delta x}{\lambda f}\right) N D
\end{aligned}
$$

where, $\Delta x$, is the distance between the output fibers, $\Delta n$ and $\Delta \lambda$ are the minimum variation in the hologram period and in the input fiber wavelength to reach the output fibers location, respectively.

In the next section, some guidelines for designing holograms by computer are described.

\section{Computer generated holograms (CGH's) design}

Taking into account the considerations made in Martín Minguez and Horche (2006), the relationships between the hologram and its Fourier Transform (F.T.) function are:

$$
\begin{aligned}
& \text { Hologram } \rightarrow \text { F.T. } \rightarrow \text { Diffraction target } \\
& \text { Diffraction target } \rightarrow \text { F.T. }{ }^{-1} \rightarrow \text { Hologram }
\end{aligned}
$$

In order to implement the $\mathrm{CGH}$, holograms are calculated by using a program based on a variation of the widely adopted simulated annealing optimization algorithm (Dames et al. 1991) which cost function to minimize the calculation error is: 


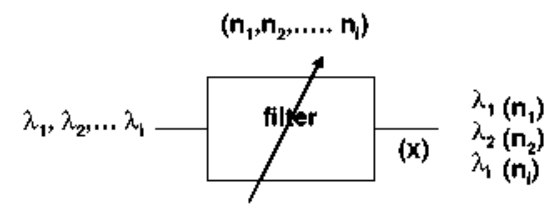

(a) Tunable Filter application:

$n$ variable; $x$ fixed

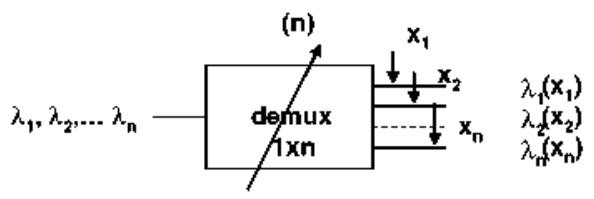

(b) Tunable Demux application: $n$ fixed; $x_{i}$ variable

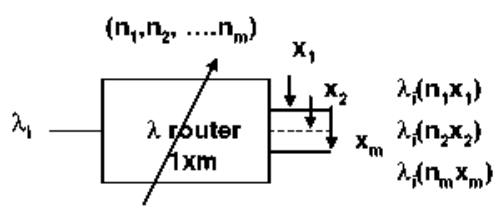

(c) Tunable $\lambda$ Router application: $n_{i}, x_{i}$, variable

Fig. 2 Basic holographic applications: a tunable filter, b tunable demultiplexer and e tunable $\lambda$ touter

$$
C=\sum_{i} \frac{\left(I_{i}^{2}-A^{2}\right)^{2}}{A_{i}^{2}}
$$

where $I_{i}^{2}$ is the calculated spot intensity for the diffraction order $i ; A_{i}^{2}$ is its defined intensity and $A^{2}$ is the average (uniform) intensity for the diffraction target spots; $t$ is the process temperature.

There are three steps in a CGH design process:

1. Diffraction Target definition: the target is the diffraction pattern that can be obtained by the SLM. Depending on the use, filter, switch or others, this target is usually an array or a matrix of spots. This is the input for the program.

2. Fourier Transform calculation: the program calculates the inverse Fourier Transform (F.T. $)^{-1}$ of the target. The optimization algorithm compares the FT of the hologram with the defined target improving at each calculation time the efficiency. Hologram pixels are flipped between the amplitude values 0,1 (or phase $0, \pi$ ) to reduce an error function, (5), specifying the difference between the desired target in the Fourier plane and the reconstruction carried out by the current state of the hologram, improving the efficiency at each calculation. The efficiency, $\eta$, is defined as : $\eta=\Sigma m$ orders diffracted light total incident light.

3. Finally, the CGH implementation in an optical substrate, like a photographic film or SLM.

The CGH designed for this work is a black \& white bars pattern implemented onto a Spatial Light Modulator, where there are only two possible states: " 1 " for white (total transparency or $\pi$ phase shift) and " 0 " for black (total darkness or 0 phase shift). Figure 3 shows the original diffraction target (a), an array of spots with different light intensities (non uniform, like in Fig. 4a), and three consecutive holograms (b, c, d), calculated by the program canying out the inverse FT according to the algorithm efficiency. A $45 \%$ of efficiency is the initial calculation value and closed to $90 \%$ efficiency is practically the best result in the optimization process.

During this calculation, the program can find out different holograms which match the diffraction target. It is possible to change, dynamically, the initial conditions (original 


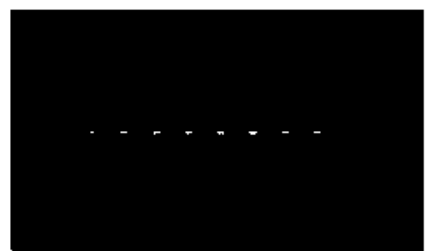

(a) Diffraction target

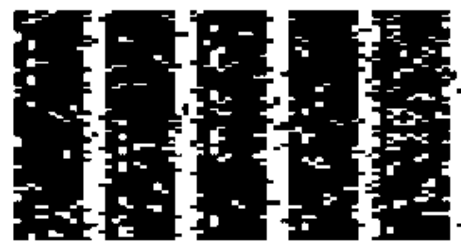

(c) Hologram: $\eta=70 \%$

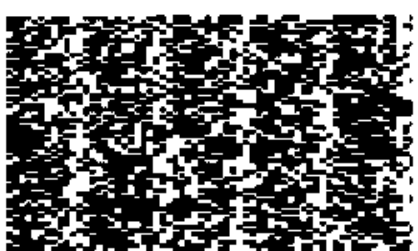

(b) Hologram: $\eta=45 \%$ eff
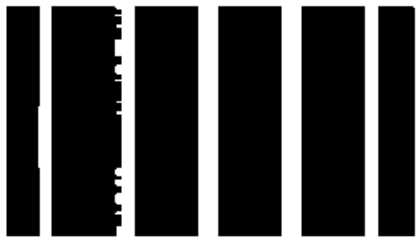

(d) Hologram: $\eta=90 \%$

Fig. 3 Hologram calculation process according to the algorithm efficiency $\eta$ : a difftaction tatget; b. c and d are calculated bolograns with $\eta=45.70$ and $90 \%$, respectively

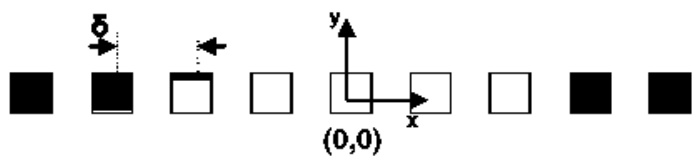

$m=-4 \quad m=-3 \quad m=-2 \quad m=-1 \quad m=0 \quad m=+1 \quad m=+2 \quad m=+3 \quad m=+4$

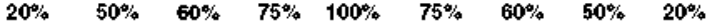

(a) Original diffraction target for hologram

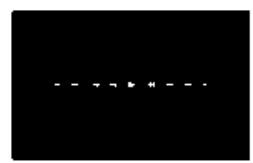

(b) Original

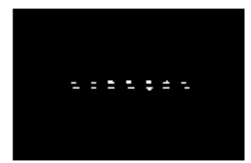

(c) Calculated

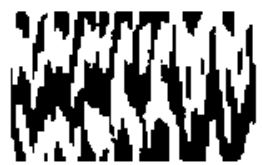

(d) Hologram

Fig. 4 a "Zoom" of the original diffraction target, b original shifted difftaction pattern along the $y$ axis, c calculated diffraction target and $\mathbf{d}$ calculated bologram when the original pattern is shifted

diffraction target and efficiency, optimization process parameters...), to modify the direction for the optimization process allowing the algorithm to escape from a local minimum and reaching the correct hologram.

The computer calculations are very sensitive to the geometrical distribution of the original diffraction target. A very light misalignment on it can produce a hologram completely different of the correct one. This effect is shown in Fig. 4 when the original array of spots (Fig. 4a) is shifted a $30 \%$ of spots distance $\delta$, (Fig. 4b), along the vertical axis $y$; the calculated target (Fig. 4c) is an array of spots "duplicated" and "shifted" instead of a singular one.

To avoid small misalignments, along the $x$ axis of the output fibers array position, with impact in the efficiency, we can optimize the hologram pattern introducing an offset in the bars position to correct them (Crossland et al. 2000). An offset of $5 \%$ of the hologram period would impact the efficiency up to a $40 \%$. 
Table 1 Holographic device parameters for CWDM/DWDM filter application

\begin{tabular}{lll}
\hline CWDM & & DWDM \\
\hline $1,270-1,590$ & $\Delta \Lambda(\mathrm{nm})$ & $1,510-1,590$ \\
1 & $\mathrm{BW}(\mathrm{nm})$ & 1 \\
1.431 & $\lambda_{0}(n m)$ & 1,551 \\
5.04 & $N . D(\mathrm{~mm})$ & 5.04 \\
25.00 & $f(\mathrm{~mm})$ & 25.00 \\
11.499 & $x(\mathrm{~mm})$ & 12.463 \\
24.71 & $\Phi(\circ)$ & 26.51 \\
1,591 & $\lambda_{\max }(\mathrm{nm})$ & 1.591 \\
1.311 & $\lambda_{\min }(\mathrm{nm})$ & 1,531 \\
\hline
\end{tabular}

\section{Holographic WDM devices description and simulation: basic structures}

\subsection{Tunable holographic filter}

In order to design a tunable holographic filter with a $-3 \mathrm{~dB}$ pass band width, $B W$, of $1 \mathrm{~nm}$ $(125 \mathrm{GHz})$, for each wavelength channel tuned, we take $d=3.5 \mathrm{\mu m}$ for the spatial period of the fixed grating. To use the same device for CWDM/DWDM, a SLM with a value of $N=720$ and $D=7 \mu \mathrm{m}$ for the spatial period, is chosen. The output singlemode fibers used in our device have a core diameter, $\Phi$ of $9 \mu \mathrm{m}$. Then, from Martín Minguez and Horche (2005) expression (5), $f$ must be greater than $23.9 \mathrm{~mm}$. As a practical value we take $f=25 \mathrm{~mm}$.

Table 1 summarizes the filter values for CWDM systems applications where channels are allocated between $\lambda_{\min }=1,310$ nmand $\lambda_{\max }=1,590 \mathrm{~nm}$, with central wavelength $\lambda_{0}=$ $1,431 \mathrm{~nm}$, and for DWDM systems $\left(\lambda_{\min }=1,530 \mathrm{~nm}\right.$ and $\lambda_{\max }=1,590 \mathrm{~nm}, \lambda_{0}=1,551$ $\mathrm{nm})$.

The operation as a tunable CWDM/DWDM filter is obtained by changing the hologram period, $n$. From the output fibers ( $F 1$ to $F 10$ ), a CWDM tunable filter uses the $F 4$ and a DWDM filter uses the $F 8$ fiber, (see Fig. 1a).

The simulation of a tunable holographic filter, from a transmission performance point of view, is shown in Fig. 5. This is another novel approach realized in this paper. For each value of the hologram, $n i$, a tuned wavelength, $\lambda i,(i=1,2,3,4)$, is obtained. The correspondence between the physical blocks of the Fig. 1a) and the simulated ones of the Fig. 5 , is the following:

- SLM and fixed grading assembly: 2nd order optical Bessel filters for tuning the output wavelengths, $\lambda i$

- lens and SLM holograms: diffraction and selection elements to choose the correct hologram, $n i$

- filter losses: are distributed among the different blocks (Martín Minguez and Horche 2005): diffraction elements, $<5+5<10 \mathrm{~dB}$ and filter losses, $<2 \mathrm{~dB}$

Figure 6 shows a simulation of the wavelength response of the holographic filter. For wavelengths close to the central, the filter response is very similar to the Gauss filter (5); for wavelengths far from the central, the filter response is similar to the 2 nd order Bessel filter (7) with less out band attenuation. Both of them have a lineal phase characteristic, which means, a constant group delay. These simulations are in agreement with experimental measurements shown in Parker et al. (1998). 


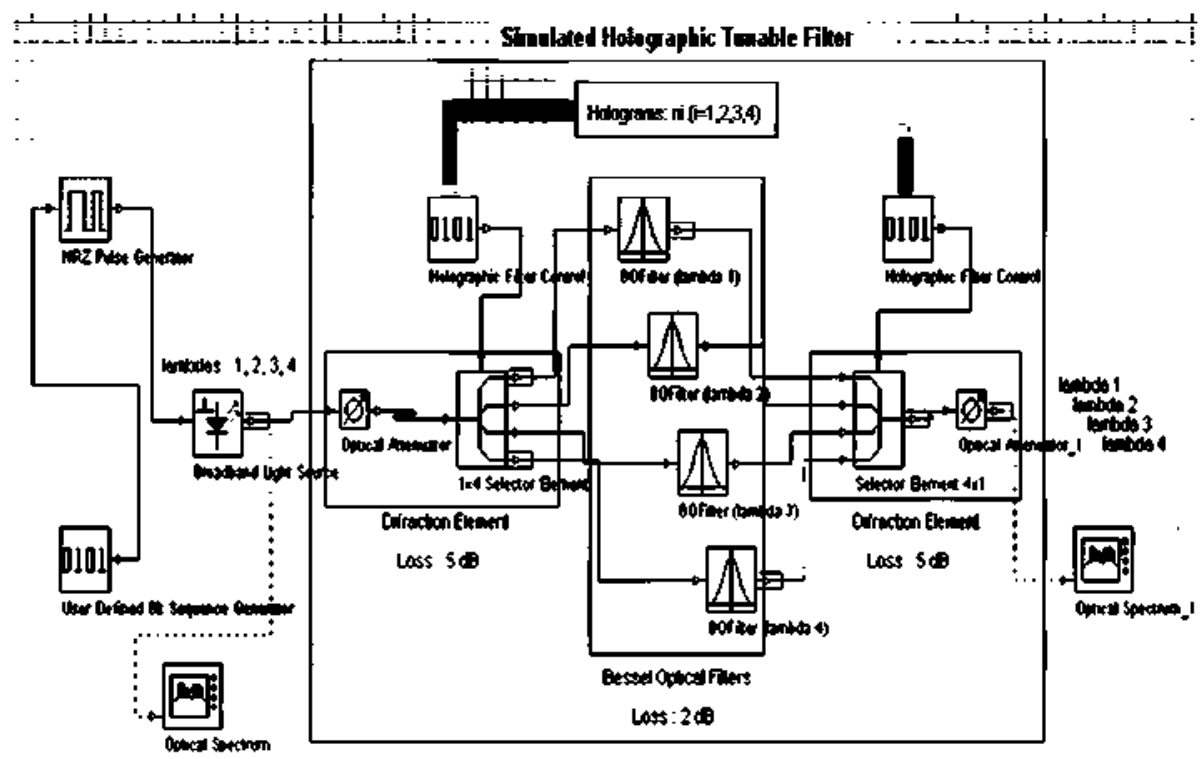

Fig. 5 Simulation of a tunable Holographic Filter

$$
\begin{aligned}
H f_{\text {Gauss }} & =1 /(\sqrt{2 \pi} \cdot B W) \cdot e^{-\left(\lambda-\lambda_{0}\right)^{2} / 2 B W^{2}}, \lambda_{0}-B W / 2<\lambda<\lambda_{0}+B W / 2 \\
H f_{B E S S E L} & =3 /\left[\left(\lambda-\lambda_{0}\right)^{2}+3\left(\lambda-\lambda_{0}\right)+3\right], \lambda<\lambda_{0}-B W / 2, \lambda>\lambda_{0}+B W / 2
\end{aligned}
$$

\subsection{Wavelength division demultiplexer}

We can use this device as a $1 \times M$ demultiplexer, where $M$ is the output fibers number. For this, a fixed value of $n$ is used and the output fibers are located in at different $x$ position. Output fibers $(9 / 125 \mu \mathrm{m})$ must be placed in agreement with the diffracted angles $\Phi$, according to input wavelengths and they have to be separated at least $\Delta x=125 \mu \mathrm{m}$.

From (4), we can calculate the $\Delta x$ taking the value of center to center wavelength channel separation, $\Delta \lambda$, into account; for CWDM systems $\Delta x=321 \mu \mathrm{m} \rightarrow \Delta \lambda=40 \mathrm{~nm}$ and for DWDM systems $\Delta x=161 \mu \mathrm{m} \rightarrow \Delta \lambda=20 \mathrm{~nm}(*)$.

In order to design a compatible device with the frequency grid provided in ITU-T G.694.1/G.694.2 Recs. for CWDM/DWDM systems, a $1 \times 4$ demultiplexer $(M=4)$ for DWDM with $\Delta \mathrm{x}=161 \mu \mathrm{m}$ and a $1 \times 8$ demultiplexer $(M=8)$ for CWDM with $\Delta x=321 \mu \mathrm{m}$, can be performed.

Table 2 summarizes the fiber position in order to select the wavelengths used in the CWDM/DWDM systems. CWDM system uses F1, F2, F3, F4, F5, F6, F8 and F10 and DWDM uses F7, F8, F9, and F10 output fibers.

(*) In this case, $\Delta \lambda$ is $<20 \mathrm{~nm}$ are not feasible due to the physical dimension of the device (i.e. $\Delta \lambda=2 \mathrm{~nm}$ and $\Delta x=161 \mu \mathrm{m} \rightarrow f$, focal distance of the lens, $=25 \mathrm{~cm}$ ).

Figure 7 shows the simulation of a tunable holographic demultiplexer. The blocks are analogue to the filter simulation, but in this case, the demultiplexed wavelengths, $\lambda i$, are obtained at different position of the output fibers $F i(i=1,2,3,4)$, for the same hologram.

In the Fig. 8 the wavelength response of the demultiplexer is pointed out for 4 wavelengths, $\lambda_{1}=1,491 \mathrm{~nm} ; \lambda_{2}=1,51 \mathrm{Inm} ; \lambda_{3}=1,53 \mathrm{Inm} ; \lambda_{4}=1,55 \mathrm{Inm}$. The attenuation between 2 

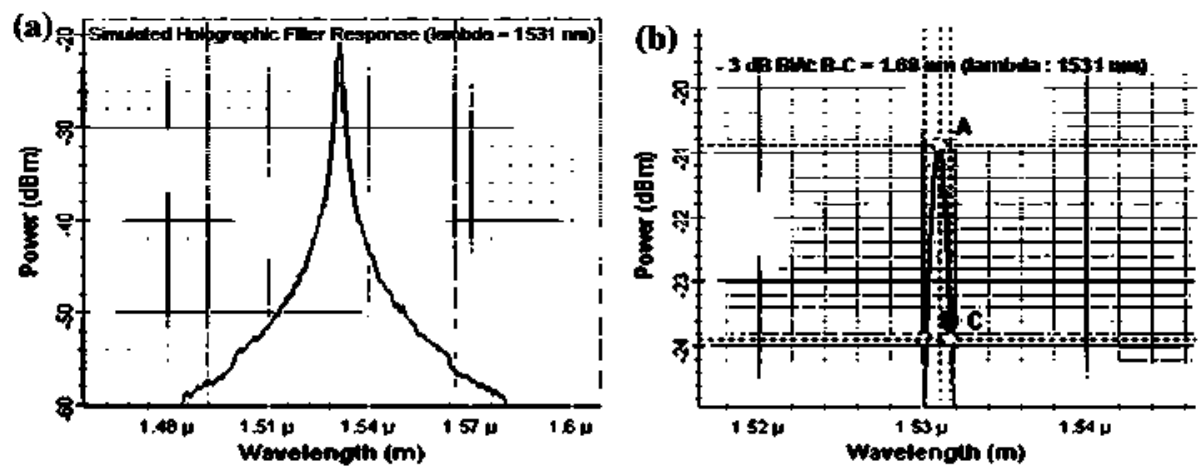

Fig. 6 a Wavelength response of the simulated Holographic Filter $\left(\lambda_{0}=1.531 \mathrm{~nm}\right)$; b central wavelength region view

Table 2 Holographic device parameters for denultiplexers/routers application

\begin{tabular}{lllll}
\hline & & Denux & \multicolumn{2}{c}{$\begin{array}{c}\text { Router } \\
n \text { value }\end{array}$} \\
\cline { 4 - 5 } Output fiber & $x(\mu \mathrm{m})$ & $\lambda(\mathrm{nm})$ & $\lambda_{0}=1,431 \mathrm{~nm}$ & $\lambda_{-0}=1,551 \mathrm{~nm}$ \\
\hline$F_{1}$ & & 1.311 & 44 & \\
$F_{2}$ & 10.535 & 1,351 & 89 & \\
$F_{3}$ & 10.856 & 1,391 & 135 & \\
$F_{4}$ & 11.178 & 1.431 & 180 & \\
$F_{5}$ & 11.499 & 1,471 & 225 & 159 \\
$F_{6}$ & 11.821 & 1.511 & 270 & 180 \\
$F_{7}$ & 12.142 & 1,531 & & 201 \\
$F_{8}$ & 12.303 & 1.551 & 315 & 222 \\
$F_{9}$ & 12.463 & 1.571 & & \\
$F_{10}$ & 12.624 & 1,591 & 360 & \\
\hline
\end{tabular}

adjacent channels, $A B$, is $>30 d B$ (worst case for CWDM, where $\Delta \lambda=20 \mathrm{~nm}$ ). The optical spectrum of the Fig. 7 is located at the Fiber 3 output $\left(\lambda_{3}=1,531 \mathrm{~nm}\right)$.

\subsection{Wavelength router}

Maintaining the output fibers in the same place, as showed in Table 2, if $n$ value (type of hologram) is properly varied, a certain wavelength coming from the input fiber can be routed to any one of the output fibers. As an example, Table 2 highlights the $n$ values for routing $\lambda_{0}=1,43 \mathrm{Inm}$ (CWDM) and $\lambda_{0}=1,551$ (DWDM) towards an output fiber; this values have been calculated from (5), by considering the variation of $n$ according to $\Delta x$. For $\Delta x=$ $161 \mu \mathrm{m}, \Delta n$ was calculated by using ( 5 ) resulting $\Delta n=21$ and for $\Delta x=321 \mu \mathrm{m}, \Delta n=45$. Therefore, the device is a $1 \times 8 \lambda$ router in case of CWDM and $1 \times 4 \lambda$ router for DWDM systems (Crossland et al. 2000).

It is necessary to highlight that the position of the fibers is compatible with all applications and that the crosstalk resulting from high-order diffraction beams ( $m= \pm 2, \pm 3$,) is outside of the location of the output array fibers, because of $\Delta \Phi \approx 4^{\circ}$, Ahderom et al. (2002). 


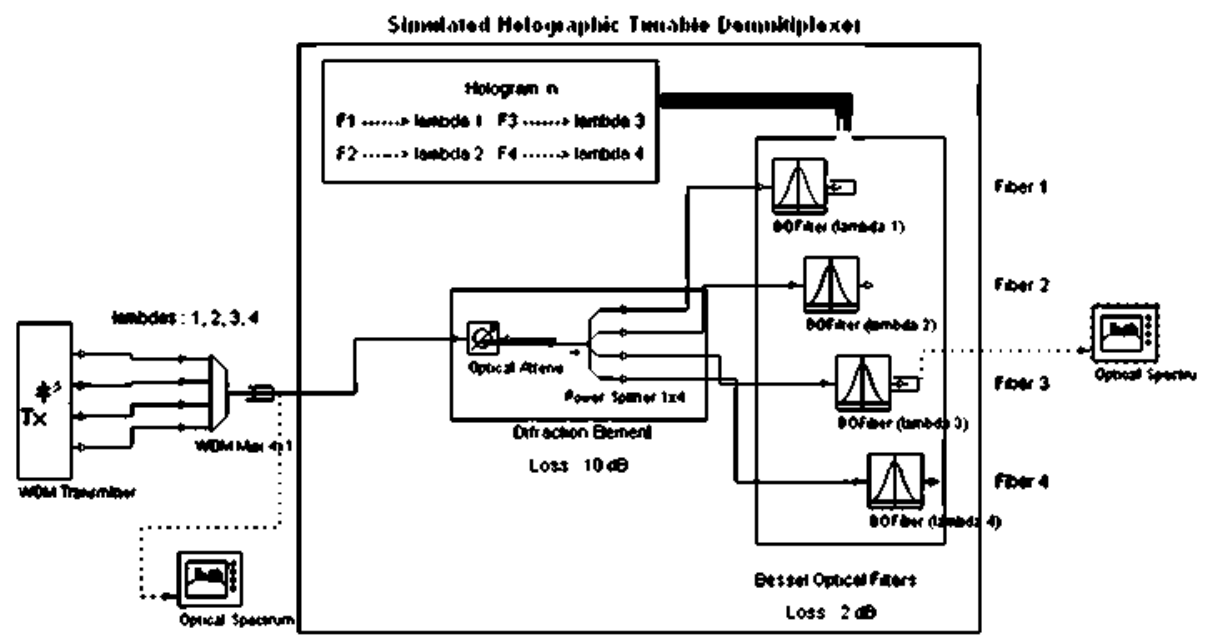

Fig. 7 Simulation of a tunable Holographic Demultiplexer
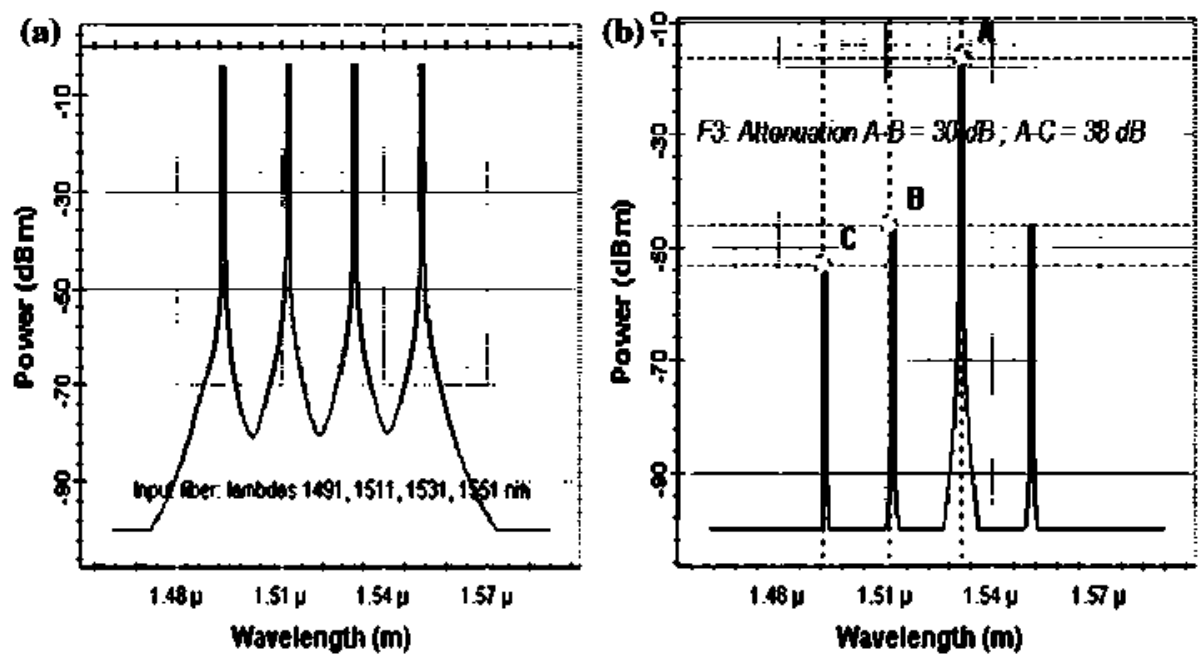

Fig. 8 Wavelength response of the Holographic Denultiplexer. a input wavelengths $\left(\lambda_{1}=1,491, \lambda_{2}=\right.$ $\left.1,511, \lambda_{3}=1,531, \lambda_{4}=1,551 \mathrm{~nm}\right) ;$ b F3 output

Figure 9 shows the simulation of a tunable holographic $\lambda$ router. Its blocks are also analogue to the filter and demultiplexer simulations, but in this case, the routed wavelength, $\lambda i$, is addressed to a different output fiber $F i(i=1,2,3,4)$, according to the hologram $n i$ selected, as it can be observed in Fig. 10 for $\lambda_{3}=1,531 \mathrm{~nm}$.

\section{Holographic WDM devices description: composed structures}

Different composed tunable holographic devices, like OADMs and optical switches, can be performed by combining the former basic structures with other devices, like conventional 


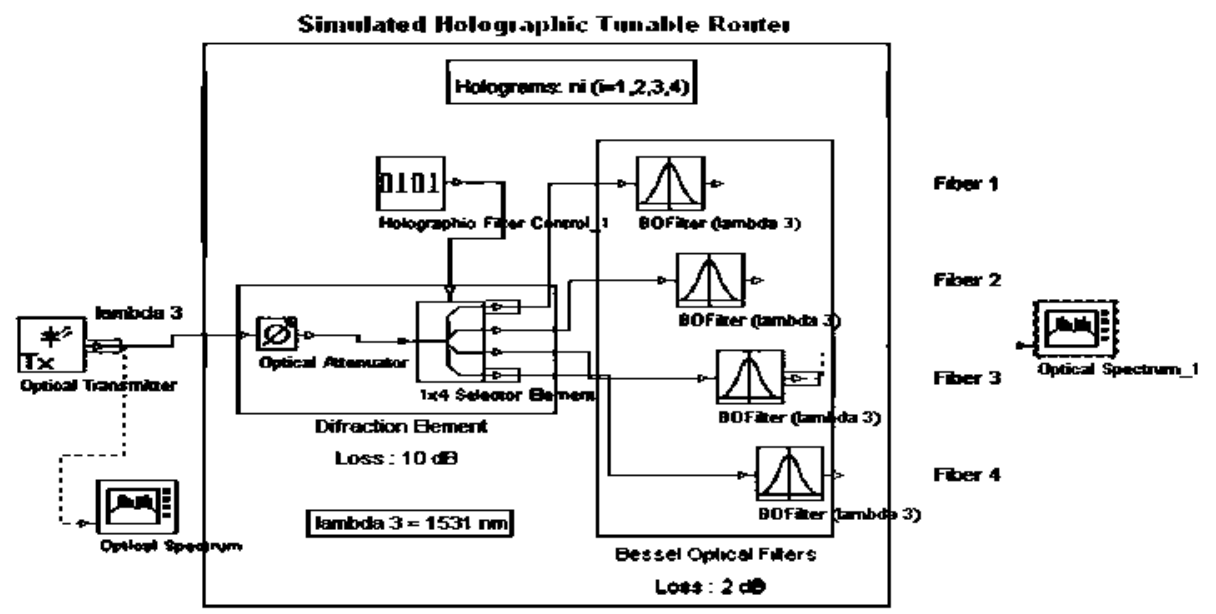

Fig. 9 Simulation of a tunable Holographic Wavelength Router
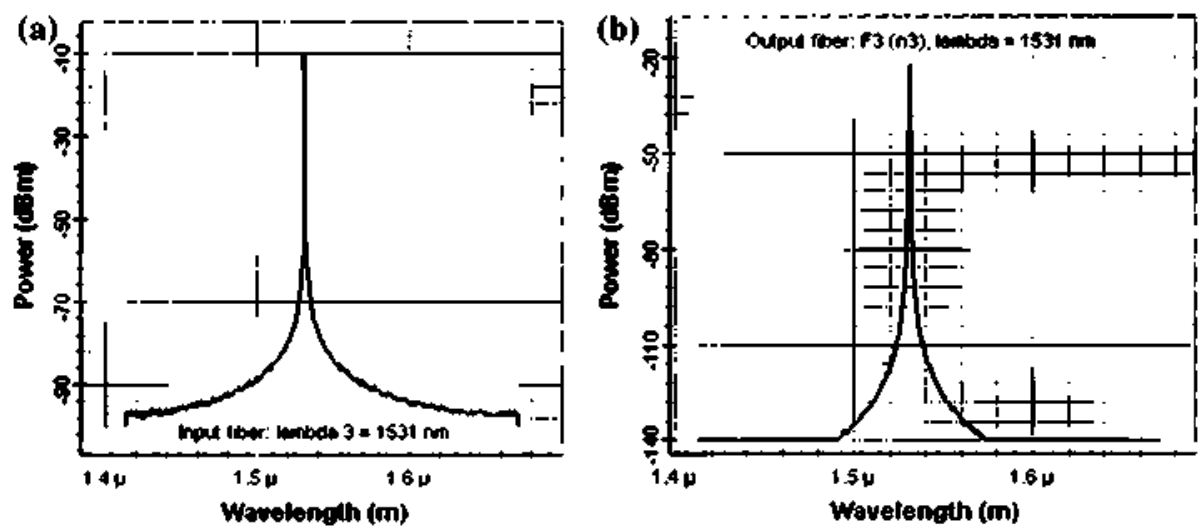

Fig. 10 Wavelength response of the Holographic Router $(\lambda .3=1,531 \mathrm{~nm})$ : a input fiber and b F3 output fiber

fixed de/multiplexers based, for example, in Bragg grating filters (Sadot and Boimovich 1998).

In the following, some examples of these configurations are pointed out; in all of them the basic holographic device is a $\lambda$ router $1 \times M$ working in a wavelength range from 1,270 to $1,610 \mathrm{~nm}$; therefore, in this case, both CWDM/DWDM systems can be addressed.

The generic tunable composed device follows the structure: fixed WDM multiplex + tunable holographic $\lambda$ router $\mathrm{I} \times M+$ fixed WDM demultiplexer; according to the application, the fixed WDM multiplexer could be removed if a single wavelength is applied to the input.

Figure 11 shows an optical tunable ADM/Switch $2 \times 2$ where two $\lambda$ routers $1 \times 2$ and two fixed mux $2 \times 1$ are interconnected. Different output wavelengths combination, in this case four $\left(2^{2}\right)$, are obtained according to the $\lambda$ router hologram type (nij). This device can work as Optical ADM or Optical Switch according to the application, taking into account that the two outputs are complementary.

Other composed structures are possible as it is commented in Martín Minguez and Horche (2006); one of them is dealing with the case where the optical input has several $\lambda$ 's, i.e., 2 


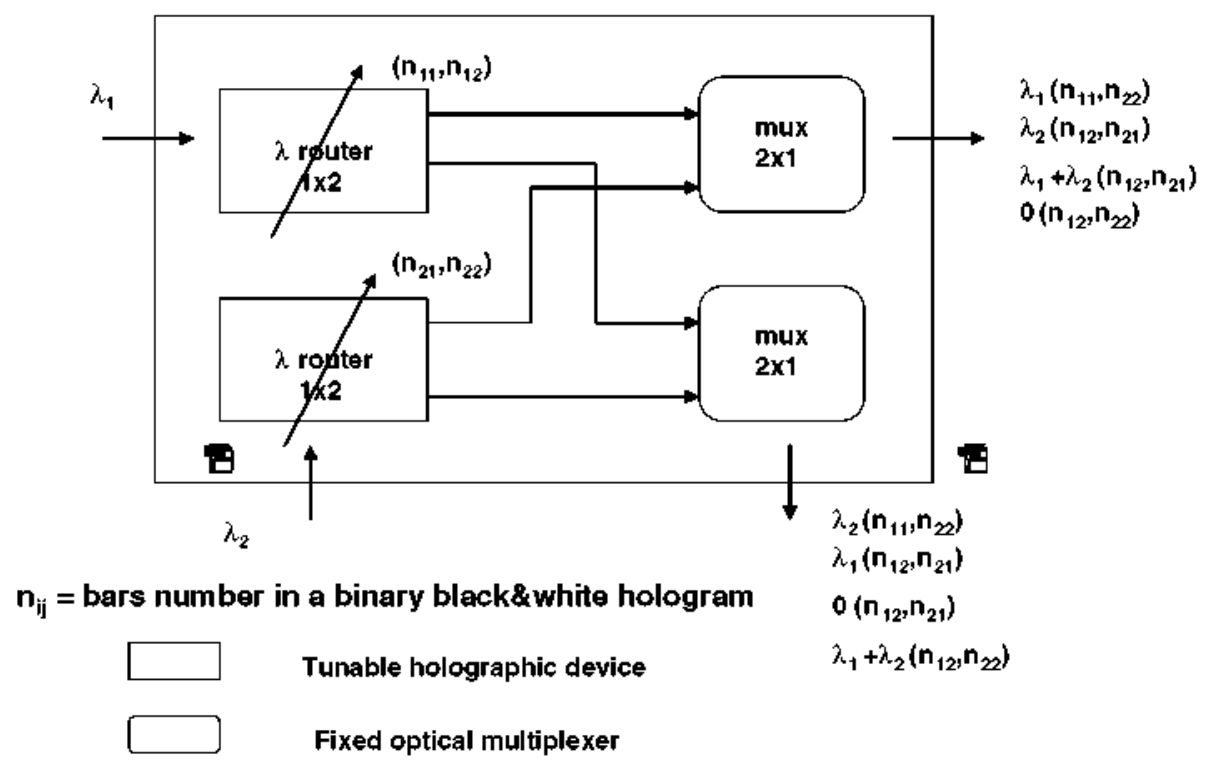

Fig. 11 Optical tunable ADM/Switch $2 \times 2$

wavelengths. To perform a complete ADM/Switch, a $1 \times 2$ demultiplexer is added to the former device; in this way any combination of the input wavelengths is addressed to the output, in total $8\left(2^{3}\right)$. The drawback of this configuration is the addition of the demultiplexer insertion losses to the final transmission path.

Other structure is a Multiplexer/Switch $4 \times 2$, composed by $4 \lambda$ routers $1 \times 2$ and $2 \mathrm{mul}$ tiplexers $4 \times 1$, where also the 2 outputs are complementary; 16 different combinations of $\lambda i(i=1,2,3,4)$ are possible at each output. This device performs a twofold function, as a multiplexer and as a switch, by reducing the number of fibers at the output.

Finally, the device of the Fig. 12 is a full Switch $4 \times 4$ matrix with 64 output combinations, at each output, according to the holograms stored on the $\lambda$ routers. One way to serialize the four $\lambda$ inputs is to place a demultiplexer $(1 \times 4)$ before the $\lambda$ routers.

All these tunable holographic devices have different applications in Metro and Access networks as it is described in the following sections.

\section{Performance parameters}

In the following, some performance parameters are described for this type of devices; these parameters are the reference to evaluate the adaptation of the devices for networking applications. In Ma and Kuo (2003) there is a complete comparison of several switching technologies according to different criteria:

- insertion loss: composed of the $\lambda$ router and de/multiplexer losses. An optimizated value for the router insertion loss is about $7 \mathrm{~dB}$ (Ahderom et al. 2002), independent of the outputs number; typical value for the de/multiplexer losses is less than $3 \mathrm{~dB}$, so, the total loss would be less than $10 \mathrm{~dB}$. 


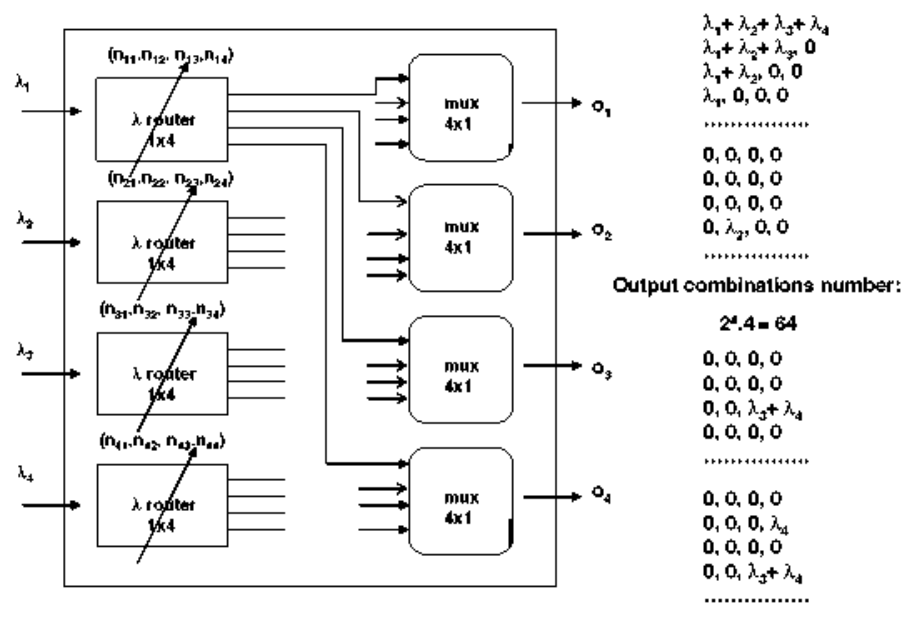

Fig. 12 Full Switch $4 \times 4$ Matrix

- switching time: due to the SLM optical switching time; it was estimated to be $250-300 \mu \mathrm{s}$, as the sum of the electric storage and FLC material response times, Horche and González (2001).

- crosstalk: in the holographic devices, due to the position of the fibers, the crosstalk resulting from high-order diffraction beams $(\mathrm{m}= \pm 2, \pm 3$, .) is outside of the location of the output array fibers $\left(\Delta \Phi<4^{\circ}\right.$ ). Crosstalk less than $-40 \mathrm{~dB}$ is a typical value (Holmes and Crossland 1994).

- polarization dependency: one of the advantages of using this technology, FLC, is its insensitivity to the light polarization for a certain range of wavelengths, allowing the increment of the transmission length.

\section{Advantages}

- multifunctional device: application as a tunable filter, demultiplexer, router, OADM or optical switch, with tunable outputs depending on the input wavelengths and holograms.

- bit rate and signal protocol independency: as an all-optical switch, not electro-optical conversion is carried out, therefore, a non electrical signal parameters dependency is reached.

- switching time: as it is few hundreds of $\mu$, the OADM/OS application in path restoration is appropriated from the service loss point of view.

- wavelength depending switching: this feature is very useful for the use of these devices as OADMs due to the additional filtering provided.

- insensitivity to the light polarization: not dynamic dispersion compensation is required to avoid transmission losses due to this characteristic.

\section{Constraints}

- losses: in composed devices it is the sum of the holographic device and the mux/ demux losses; it is necessary to optimize the design of the device to reduce them (Ahderom et al. 2002), taking into account that the losses due to the holographic part are fixed, independent of the output number.

Figure 13 shows a device composed by a Semiconductor Optical Amplifier (SOA) and a holographic wavelength router. The SOA performs the wavelength conversion by a non 


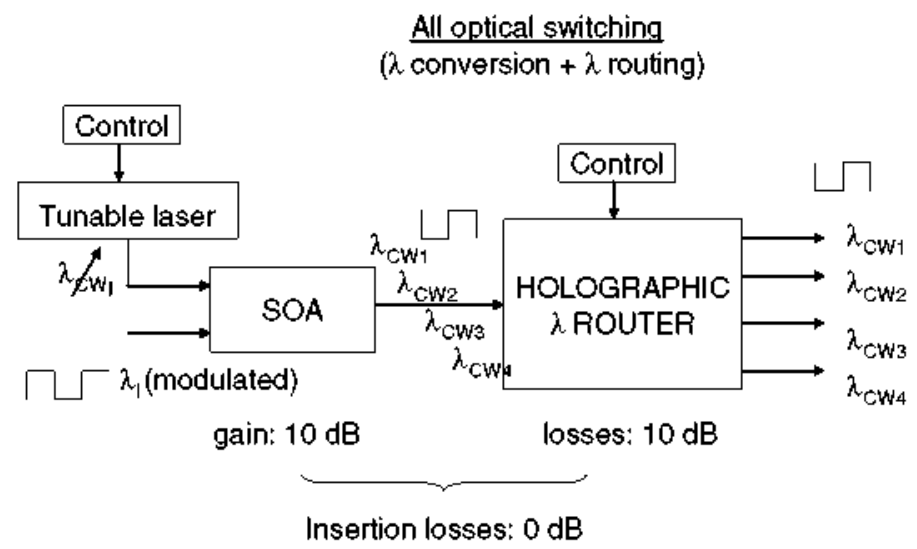

Fig. 13 Device composed by an optical $\lambda$ converter and a holographic $\lambda$ router

lineal operation using the Cross Gain Modulation (XGM) method. An incident wavelength, $\lambda i$, modulated by a digital signal is combined with the wavelength $\lambda_{C W j}$ generated by a tunable laser ( $\mathrm{CW}$ ) into the SOA. At the amplifier output, according to different $\mathrm{CWj}$ wavelengths, $\lambda_{C W_{j}}$ signals are obtained modulated with the digital signal from the incident $\lambda i$ wavelength. These $\lambda_{c w_{j}}$ signals are also amplified and inverted.

The holographic wavelength router, depending on the input signal, $\lambda_{C W_{j}}$, and the generated hologram (nij) stored in the SLM, addresses this signal to the assigned output. As it has been commented, this technology has the drawback of the high insertion losses (less than 10 $\mathrm{dB}$, using an optimized device). In order to solve this problem, by combining a SOA with the holographic router, this insertion loss is compensated with the amplifier gain in the saturation zone of operation. A parameter to control in the SOA operation, is related with the amplified spontaneous emission (ASE) because of the impact on the signal deformation.

Figure 14 shows the simulation of this device, composed of three different blocks: a CW tunable laser, a wavelength conversion semiconductor optical amplifier and a wavelength holographic router.

In Fig. 15, the response of the Wavelength Conversion and Routing Holographic Device (WCR-HD) is represented for a $2.5 \mathrm{~Gb} / \mathrm{s}$ input signal, $\lambda_{i n}=1,540 \mathrm{~nm}$, that is converted to an output signal, $\lambda_{\text {out }}=1,520 \mathrm{~nm}$, where the losses of the holographic router are compensated by the gain of the SOA.

- holograms generation and operation: for the operation of these devices it is necessary to generate holograms, usually with a computer, CGH (Computer Generated Holograms) (Dames et al. 1991), and to configure with them the SLM. To perform the switching operation a closed control between the holographic component (SLM) and the computer is needed to assign the correspondent hologram from a local database. This performance is represented in Fig. 16, where a switching control acts over the PC-SLMs interface.

\section{Application in Metro and Access optical networks}

Figure 17 shows a typical Access and Metro network configuration.

For the Metro network, the nodes are linked by a single/double ring to prevent interruptions in the service. $1 / 2.5 \mathrm{Gbit} / \mathrm{s}$ Ethernet links are usual for this application. In some 


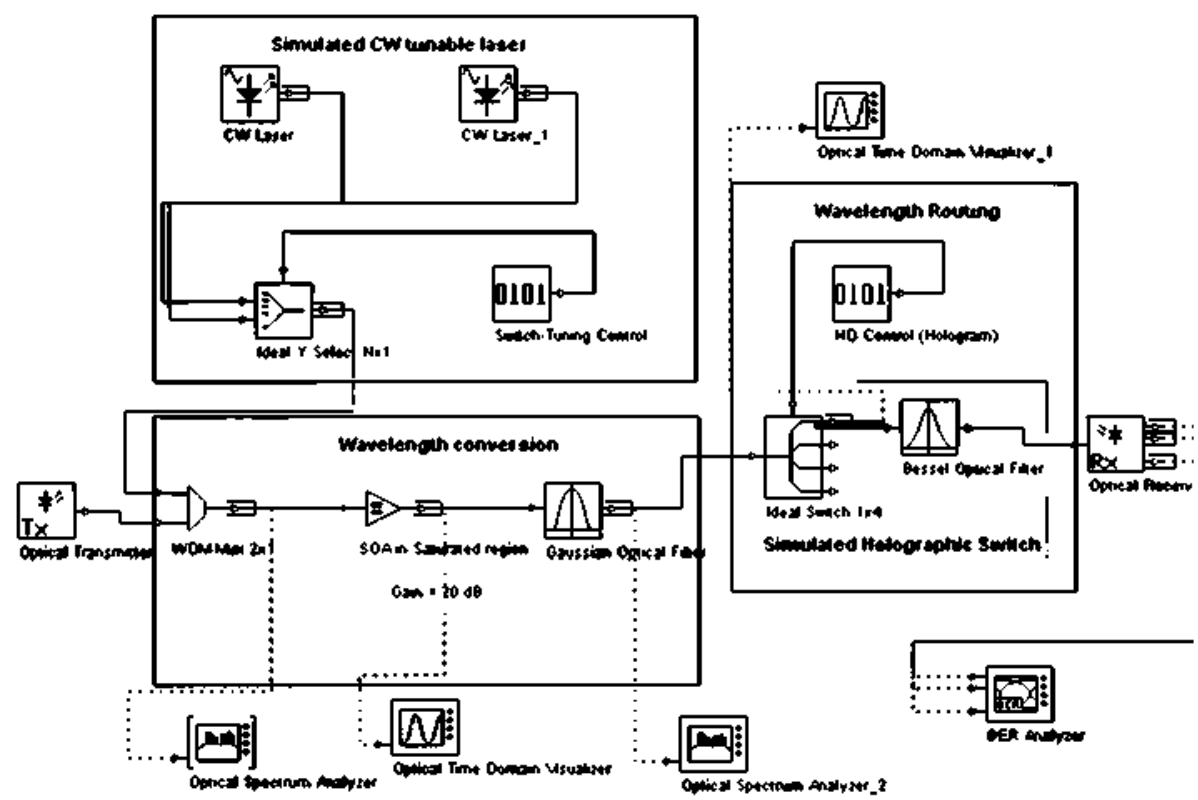

Fig. 14 Wavelength Conversion and Routing Holographic Device (WCR-HD) sinulation

cases, depending on the size of the city, several Metro networks are interconnected by optical switches with a "gateway Metro network" to communicate with the long haul network (I Gbit/s $-40 \mathrm{Gbit} / \mathrm{s})$.

Three different Access networks are drawn in the figure:

- Point to Point (P-P): with a dedicated link between the OLT (Optical Line Termination) placed at the $\mathrm{CO}$ (Central Office) and the ONT (Optical Network Termination) at the customer premises. Typical bit rates of $622 \mathrm{Mb} / \mathrm{s}$ (down) and $155 \mathrm{Mb} / \mathrm{s}$ (up) are used in this network.

- FTTO: Fiber to the Office Passive Optical Network, with an intermediate branching point or Remote Node (RN), where splitters, mux/demux, AWGs,..., are placed for addressing the services to the correspondent BONT (Business ONT). Usually, a LAN (Local Area Network) is connected to the BONT to distribute the services among the different user's terminals, acting as a hub. Typical bit rates of $2.5 / 1 \mathrm{~Gb} / \mathrm{s}$ (down) and $1,000 / 622 \mathrm{Mb} / \mathrm{s}$ (up) are used in this networks.

- FTTH: Fiber to the Home, similar to the former network, but in this case the RONT (Residential ONT) is less complex than the BONT. Triple Play services (voice, video, data) are provided by these networks where distribution services like TV, BB Internet,..., use a great quantity of bandwidth. Bit rates of $1 \mathrm{~Gb} / \mathrm{s}-622 \mathrm{Mb} / \mathrm{s}$ (down) and 622-150 $\mathrm{Mb} / \mathrm{s}$ (up) are currently used in this application.

\subsection{Tunable holographic devices application in Metro networks}

Two applications of these devices in Metro networks, Fig. 18, are commented in the following paragraphs, Riza and Yuan (1999). 
(a) Signal Power

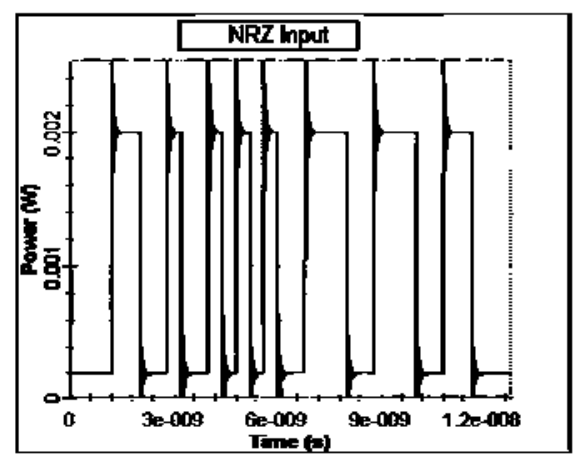

(b) Q Factor
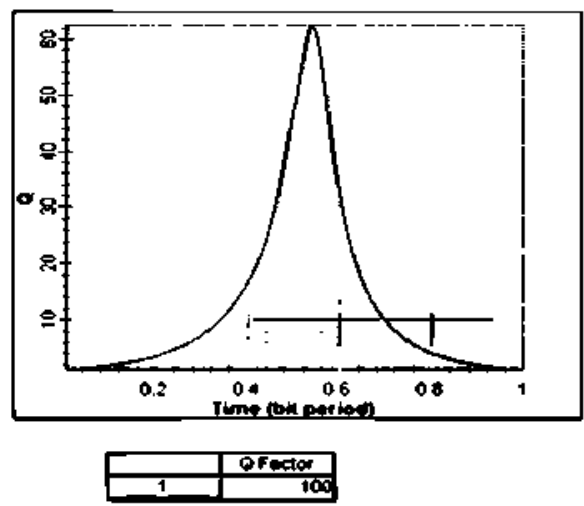

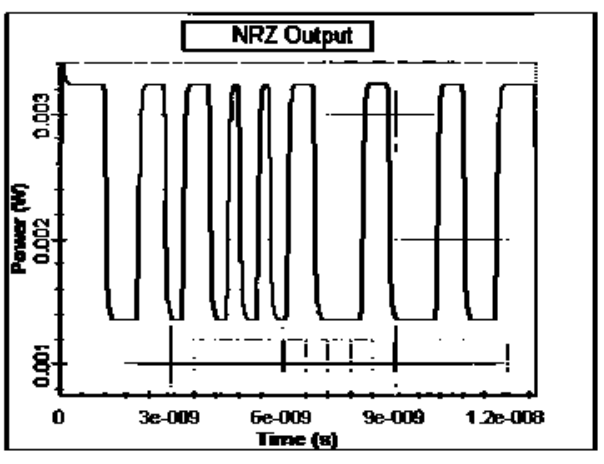

(c) Eye Diagram
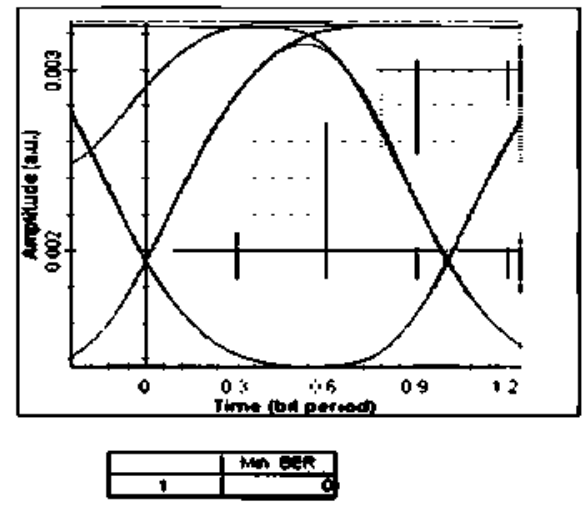

Fig. 15 WCR-HD response for a 2.5 Gbit/s input signal: a $\lambda_{-i n}=1.540 \mathrm{~nm}$, with wavelength conversion. $\lambda_{\text {-out }}=1,520 \mathrm{~nm}$. and losses conpensation, $\mathbf{b} Q$ factor $\approx 100$ and $\mathrm{c} B E R \approx 0$

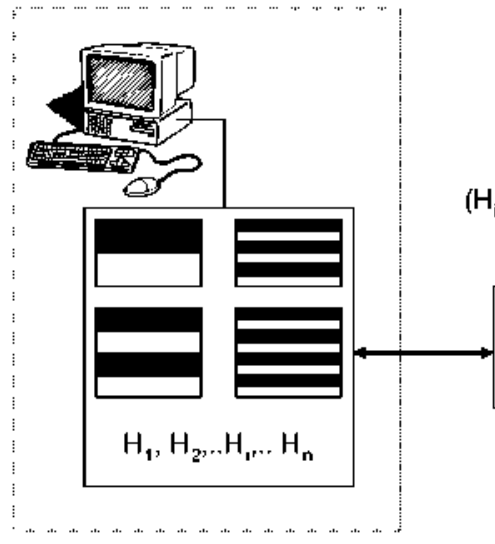

Holograms $\left(\mathrm{H}_{\mathrm{j}}\right)$ stored in the PC
$\left(\mathrm{H}_{\mathrm{i}}\right.$ assigment to the $\left.\mathrm{SLM}\right)$ according to $n_{1}$

PC-SLMs Interface

Switching control

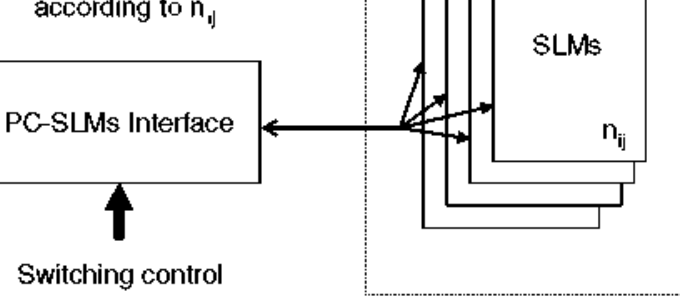

Holographic device

Fig. 16 Tunable holographic device: switching operation 


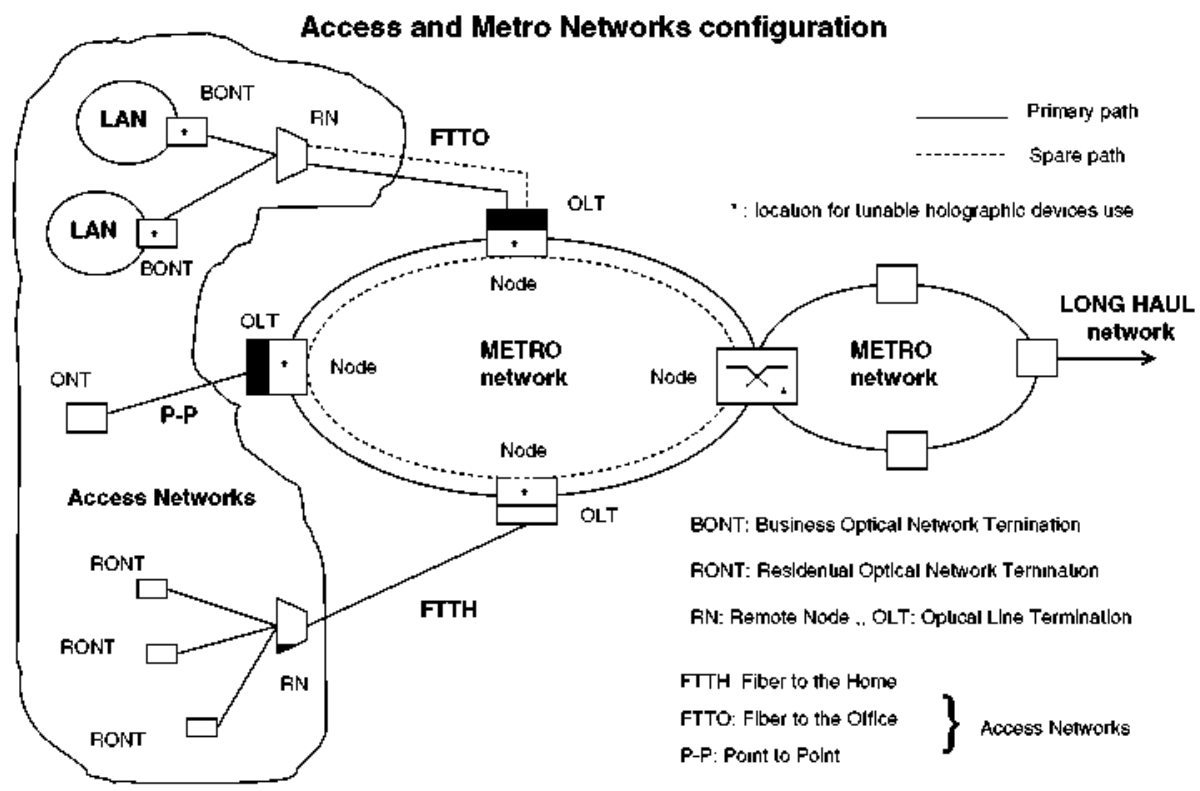

Fig. 17 Genetic Access and Metro networks

\section{Metro Networks application}

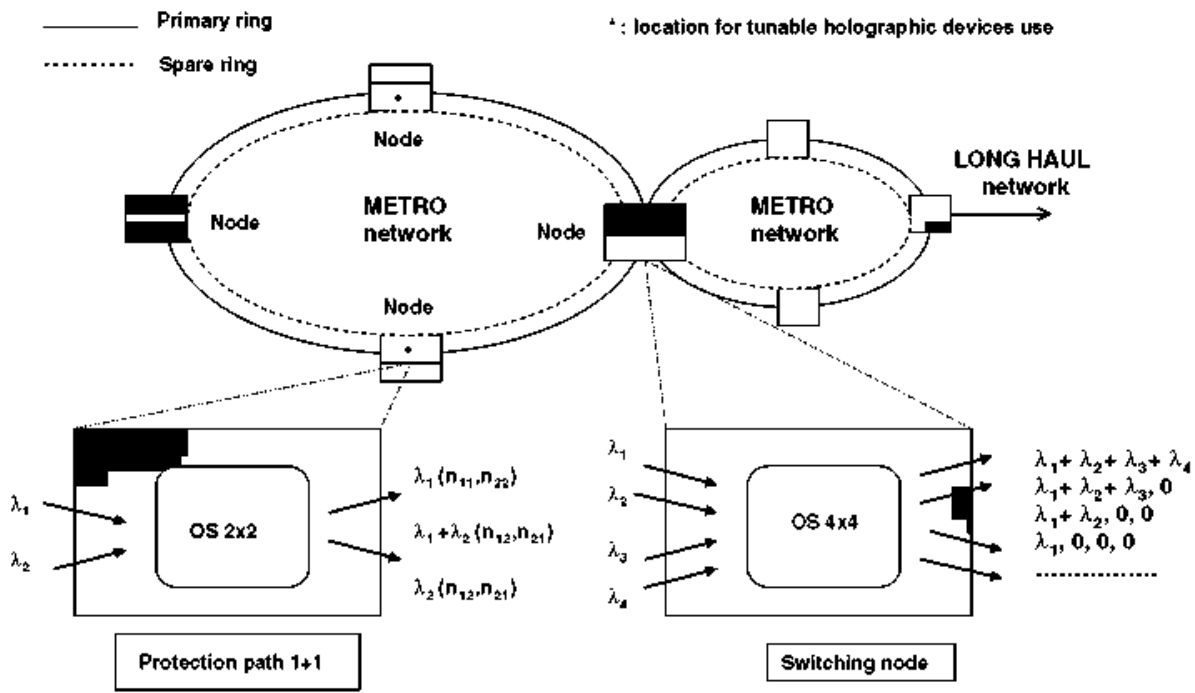

Fig. 18 Protection path $l+l$ and Switching node applications in Metro networks

The first one, an Optical ADM/Switch $2 \times 2$ is used to protect the double ring in the interconnection between nodes (Tebben et al. 2001). A failure in the primary ring $\left(\lambda_{1}\right)$, correspondent to the holograms combination $\left(\mathrm{n}_{11}, \mathrm{n}_{22}\right)$, can be solved changing, by an automatic switch control procedure on the device, to the spare holograms combination $\left(\mathrm{n}_{12}, \mathrm{n}_{21}\right)$; in 


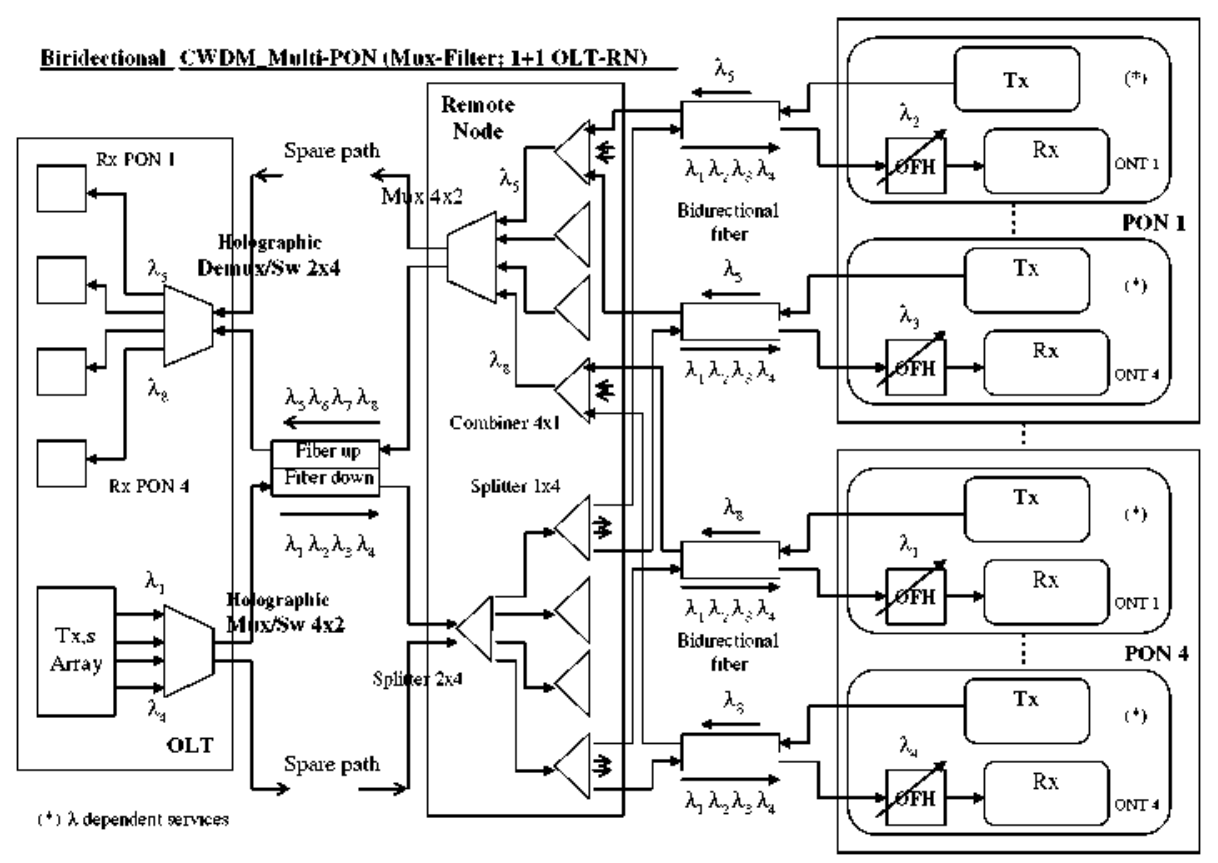

Fig. 19 Example of a FTTO Access Network configuration

this way the secondary ring $\left(\lambda_{2}\right)$ becomes active. As the switching time is about few hundreds of $\mu \mathrm{s}, \mathrm{a}$ quick path restoration could be reached.

In the second one, an Optical Switch $2 \times 2$ (or $4 \times 4$ ) can be used to interconnect two Metro rings networks for exchanging its wavelengths or to route any combination of the $\lambda_{j}$ $(1=1,2 \ldots 3,4)$ to the nodes of the rings; in this way both networks have access to the long-haul network and a full interconnectivity between them is obtained.

\subsection{Application in Access networks}

Figure 19 illustrates a generic Multi-PON FTTO network using CWDM (Coarse Wavelength Division Multiplexing) technology where 4 PONs are addressed. At the Remote Node (RN), between the OLT and the BONTs, passive elements, like splitters, combiners, multiplexers,.. are placed for interconnections with the remote network terminations, ONTs (Martín Minguez and Horche 2007b).

In each PON, an ONT has access to all wavelengths in the down direction $\left(\lambda_{1}, \lambda_{2}, \lambda_{3}, \lambda_{4}\right)$, selecting one of them by a tunable filter; with this configuration, instead of an optical demultiplexer, a splitter is placed in the RN. In the upstream direction each PON transmits in the assigned wavelength $\left(\lambda_{5}, \lambda_{6}, \lambda_{7}, \lambda_{8}\right)$ and by a multiplexer in the RN all of them are placed in the same fiber.

A bidirectional fiber between the RN and the ONTs can be used to save cost fiber installation; from the OLT to the RN two different fibers (down and up) are installed for reliability reasons (all Multi-PON traffic is going through this path).

The technical characteristics of this network are shown in Table 3 and they are in agreement with ITU G. 983.x Recommendations.

In this case also two applications are commented, see Fig. 20. In the first application, a tunable holographic filter is located at the BONT premises. This device is used to select the 
Table 3 FTTO network technical characteristics (according to ITU Rec. 983.x)

\begin{tabular}{ll}
\hline Bit rate: & Tx mean channel output power: \\
Down: $2.4 \mathrm{~Gb} / \mathrm{s}$ & OLT: $0 \mathrm{dBm}$ \\
Up: $1.2 \mathrm{~Gb} / \mathrm{s}$ & ONT: $-3 \mathrm{dBm}$ \\
Power budget $(3 \mathrm{~dB}$ of system nargin): & Rx mean channel input power: \\
Down: $20+3=23 \mathrm{~dB}$ & OLT: $-23 \mathrm{dBm}$ \\
Up: $20+3=23 \mathrm{~dB}$ & ONT: $-20 \mathrm{dBm}$ \\
Max. Distance: & Max. chromatic dispersion: \\
OLT-Branching Point: $10 \mathrm{Km}$ & Down: $1.600 \mathrm{ps} / \mathrm{nm}$ \\
Branching Point- ONTs: $15 \mathrm{Km}$ & Up: $2,000 \mathrm{ps} / \mathrm{nm}$ \\
Wavelength allocation $(C W D M):$ & \\
Down: $\lambda_{1}=1,471 \mathrm{~nm} ; \lambda_{2}=1.491 \mathrm{~nm} ; \lambda_{-3}=1,511 \mathrm{~nm} ; \lambda_{4}=1,531 \mathrm{~nm}$ \\
Up: $\lambda_{5}=1.551 \mathrm{~nm} ; \lambda_{6}=1,571 \mathrm{~nm} ; \lambda_{7}=1.591 \mathrm{~nm} ; \lambda_{8}=1.611 \mathrm{~nm}$
\end{tabular}
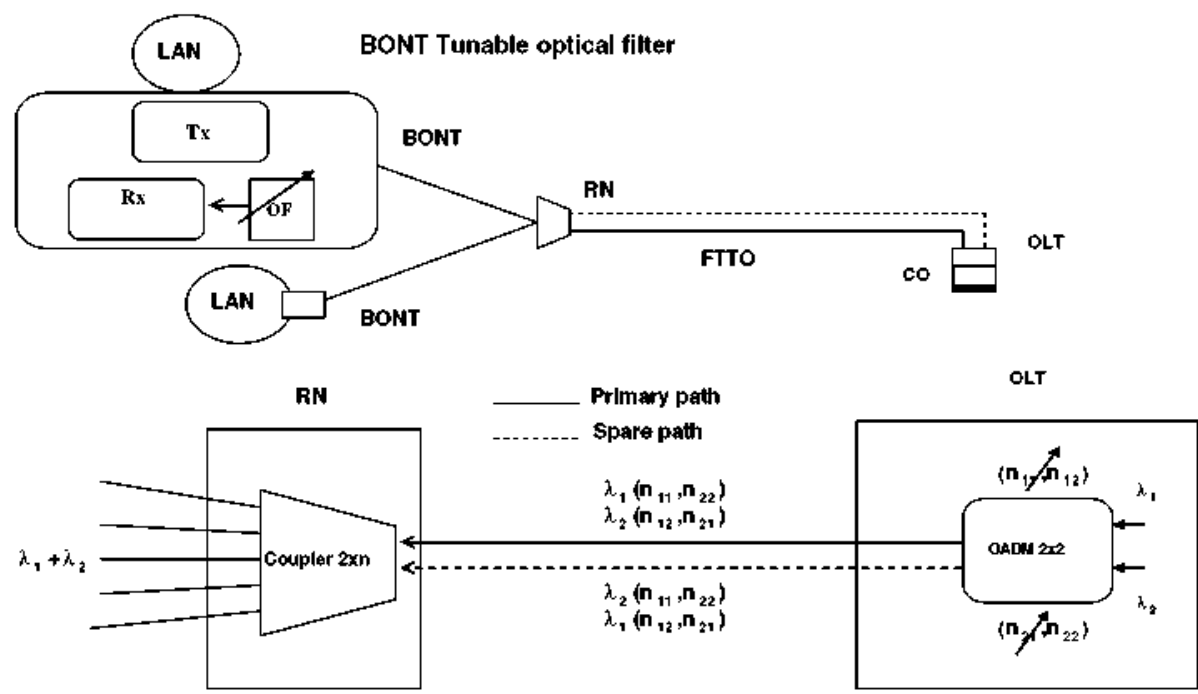

Optical path protection (between RN and OLT)

Fig. 20 BON Tunable optical filter and Protection OLT-RN path $1+1$ applications

wavelength where assigned services for the subscribers are provided; this type of feature could be useful when the BONT, due to the complexity of the LAN connected to, acts as a hub managing the subscriber premises network and the services assignment.

In the other application, an OADM $2 \times 2$ is used to protect the optical path between the OLT (Optical Line Termination) and the RN (Remote Node or branching point); in case of a fiber failure, the services can be re-routed to the spare path by switching the device from the primary working path to the spare one.

A simulation of the Holographic Mux/Switch $4 \times 2$ device, used at the OLT side in Fig. 19 , has been performed. Figure 2 la shows the configuration of the device where, the core elements are a holographic router $1 \times 2$ and an optical Bessel filter. Operation wavelengths, Fig. 21b and c, are addressed to fiber 1, 2 according to the hologram (nij) chosen. 

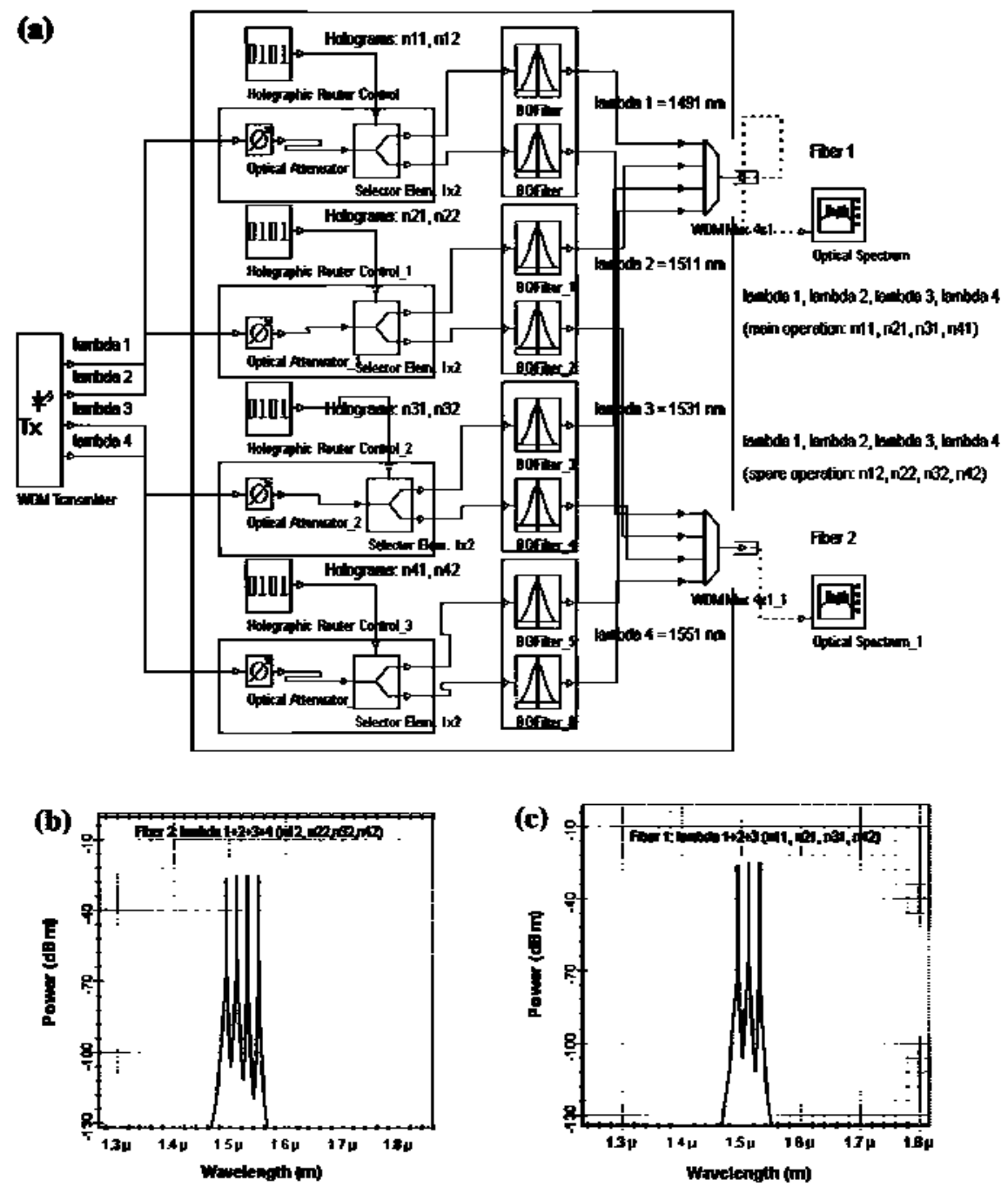

Fig. 21 a Simulated Holographic. Multiplexer/Switch 4 \% 2 structure. b and c operation tesults according to the chosen hologram (nij)

In Fig. 22a, the eye diagram at ONT side after a total distance (OLT_RN)+(RN_ONT) of $10+15=25 \mathrm{Km}$ is presented for a BER $=1 \cdot 10^{-11}$ (Fig. $22 \mathrm{~b}$ ). Values from Table 3 have been chosen regarding the bit-rate, $2.4 \mathrm{Gbit} / \mathrm{s}$ and power budget, $20 \mathrm{~dB}$. The operation wavelength is $\lambda=1,511 \mathrm{~nm}$.

\section{Comparison with other currently used technologies}

ROADMs (Reconfigurable Optical Add-Drop Multiplexers) are optical devices where holographic components can be used. Its multiple applications (multiplexers, routers, switches,...) cover a lot of configurations in optical networks. The diversity of ROADMs architectures 


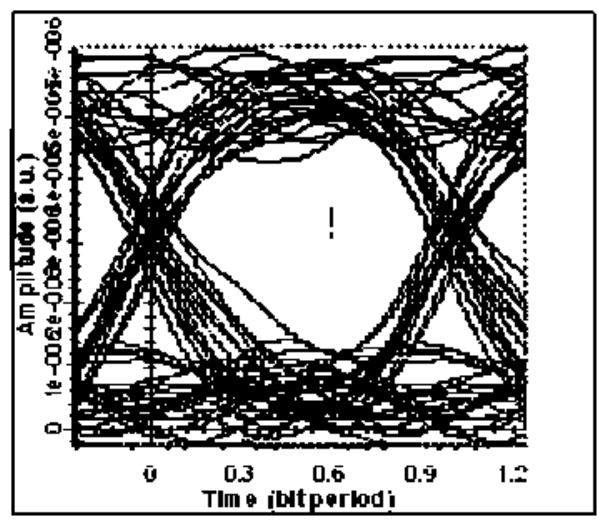

(a) Eye Diagram

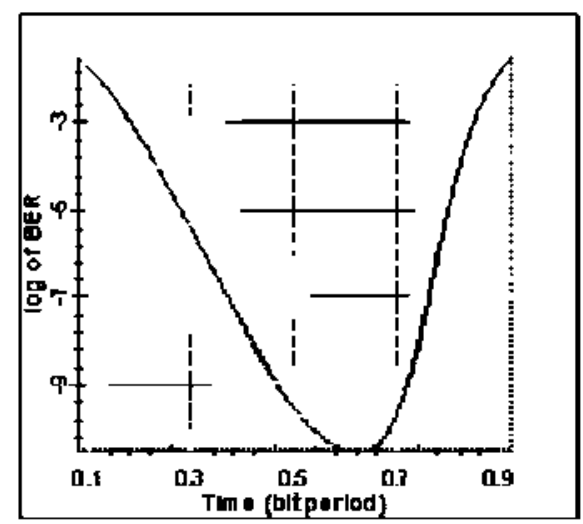

(b) Bit Error Rate (BER)

Fig. 22 Simulated Access Network performance with values of Table 3: a Eye diagram and $\mathbf{b}$ BER at the ONT side

Table 4 Wavelength selective routing technologies for ROADMs

\begin{tabular}{|c|c|c|c|}
\hline Technology & Principle of operation & Advantages & Drawbacks \\
\hline $\begin{array}{l}\text { Holographic } \\
\text { SLMs with } \\
\text { liquid crystal } \\
\text { components }\end{array}$ & $\begin{array}{l}\text { Control of light } \\
\text { diffraction by } \\
\text { holograms }\end{array}$ & $\begin{array}{l}\text { Non complex } \\
\text { implementation }\end{array}$ & $\begin{array}{l}\text { Optical performance } \\
\text { degrades for high } \\
\text { port counts due to } \\
\text { losses and } \\
\text { cross-talk }\end{array}$ \\
\hline $\begin{array}{l}\text { Micro-electrone- } \\
\text { chanical systems } \\
\text { (MEMS) }\end{array}$ & $\begin{array}{l}\text { Physical } \\
\text { displacement of } \\
\text { light using } \\
\text { mitrored MEMS } \\
\text { surfaces }\end{array}$ & $\begin{array}{l}\text { Scalable over a wide } \\
\text { range of poit sizes }\end{array}$ & $\begin{array}{l}\text { Not economical for } \\
\text { low port counts } \\
\text { due to the } \\
\text { complex } \\
\text { inplementation }\end{array}$ \\
\hline
\end{tabular}

requires different sizes and configurations of them, Homa and Bala (2008). At present, there are requirements with port counts ranging from $\mathrm{I} \times \mathrm{I}$ through $\mathrm{I} \times N,(N>10)$ with equalization of the input wavelengths power level. Table 4 provides a high-level summary of two of the main technologies currently being used for ROADMs. In the case of low port counts $1 \times 1,2 \times 1$, and $4 \times 1$, holographic liquid crystal-based design for economy and ease of manufacture can be used. For high port counts the use of a combination of MEMS (Micro-Electro-Mechanical Systems) for wavelength switching and liquid crystal for equalization is a current option.

In Martín-Minguez et al. (2010) the design of equalized holographic ROADM devices for applications in CWDM optical networks have been developed by using a mixed hologram corresponding to the combination of several input wavelengths. The tuning of a broad range of wavelengths is obtained allowing full routing of several channels from the input fiber to the outputs.

By changing the active pixels number in the SLM for each hologram, in order to maintain a fixed output power level, channel equalization can be reached. Intrinsic losses of the device have been optimized using 4-phases holograms which diffraction efficiency, for the $1^{\text {st }}$ order, is twice than binary holograms. Also, the ROADM size can be minimized by using $a$ " $2 \mathrm{f}$-folded" instead of a "lineal-4f $\mathrm{f}^{+t}$ for the optical structure. 
To reduce the total insertion losses of the holographic device and to increment the input power range for equalization, a SOA has been added. The device designed in Martín-Minguez et al. (2010) is able, with the same physical structure, to cover the complete CWDM band, $1,271-1,611 \mathrm{~nm}$, by using groups of 4 input wavelengths (separated $20 \mathrm{~nm}$ ), as it is recommended in ITU G. 695 .

\section{Conclusions}

Tunable holographic WDM devices for applications in optical networks have been studied. Based on the same holographic structure, designs of both basic and composed devices have been provided. Simulations of them, from the optical transmission point of view, have been done for applications like filters, routers or optical switches; these simulations take into account the adaptation of holographic optical components characteristics (SLM, diffraction grating, lens) to transmission component parameters (filter, attenuator, splitter) in order to use a commercial software for optical communication systems applications. In addition, short guidelines for Computer Holograms Design are provided to address some issues related to the operation of these holographic devices.

Performance parameters, like losses, switching time, crosstalk and polarization insensitivity have been analyzed. These parameters have very competitive characteristics with respect to other switching technologies; in order to reduce the insertion losses, a device optimization design has been done. Wavelength conversion and routing application using a SOA to compensate its losses, has been described.

Moreover, the use of tunable holographic WDM devices in Metro and Access networks has been analyzed. Applications in Metro networks like path protection between nodes or a switch matrix for ring networks interconnection, have been commented. Other applications in Access networks, like OLT-RN path protection or the use of tunable filters in the BONT to select wavelengths according to the type of services provided, show the versatility of these devices in this type of networks.

Ackmowledgments The authors gratefully acknowledge the support of the MICINN (Spain) throngh project TEC2010-18540 (ROADtoPON).

\section{References}

Ahderom, S. Rajsi. M., et al.: Applications of liquid crystal spatial light modulators in optical connunications. High Speed Networks and Multimedia Conmunications. 5th International Conference on. 3-5. pp. 239-242, July (2002)

Crossland, W.A. et al.: Holographic optical switching: the ROSES demostrator. J. Lightwave. Tech. 18(12), 1845-1853 (2000)

Dames. M. Dowling, R. et al.: Efticient optical elements to generate intensity weighted spot arrays: design and fabrication. Appl. Opt. 30. 2685-2691 (1991)

Holmes, MJ., Crossland, W.A.: Optimization of WDM demultiplexers. IEE-Colloquium-on- ${ }^{+}$Transparent-Optical-Networks:-Applications,-Architectures-and-Technology'-Digest No.1994/103. 1994: 8/1-7 1994

Honja, J.. Bala, K.: ROADM Architectures and Their Enabling WSS Technology. IEEE Connunnications Magazine, pp 150-153. July (2008)

Horche, P. R. González Herráez. M.: Pelformance Analysis of Dynanic Holographic Interconnects using a Ferroelectric Liquid-Crystal Spatial Light Modulator. Long-Haul and Access Networks, Optical Metro and WDM, NOC. 2001, Pp. 276-282, (2001) 
Kashnow, R.A., Bigelow, J.E.: Difftaction from a liquid crystal phase grating. Appl. Opt. 12(10), 2303$2304(1973)$

Ma. X., Kuo, G.S.: Optical switching technology comparison: optical MEMs vs. other technologies. IEEE Optical Communications S16-S23, Nov (2003)

Martín Minguez, A.: Contribución al estudio y optimización de dispositivos basados en holografía dinámica para su uso en redes ópticas pasivas multiplexadas en longitud de onda, WDM-PON. Tesis Doctoral, Universidad Politécnica de Madrid (UPM). http://oa.upm.es/1781/. Nov (2007)

Martín Minguez, A.. Horche, P.R.: Dynamic holographic wavelength filtering for CWDM applications. SPIE'05, Optics \& Photonics, paper 5907-1922, San Diego, Aug (2005)

Martín Minguez, A. . Horche, P. R.: Tunable WDM Holographic Devices: Aplication in Reconfigurable Access and Metro Networks. Procc. IET 2nd International Conference on Access Technologies, Cambridge, UK, Jun (2006)

Martín Minguez. A.. Horche. P.R.: A nultipurpose WDM holographic device for tiltering. wavelength multiplexing and tonting applications. Phot. Netw. Commun. J. 13(3), 313-322 (2007a)

Martín Minguez, A., Horche, P.R.: Application of WDM Holographic Devices in Access and Metro Networks. Optical and Quantum Electronics Journal 39(2), 131-146 (2007b)

Martín-Minguez, A.. del Rio. C., Horche, P. R.: Design of Equalized Holographic ROADMs (Reconfigurable Optical Add-Drop Multiplexers). III International Workshop on Liquid Crystals for Photonics, LCP 2010. Elche 8-10 Sept (2010)

Martin Minguez, A.. Horche, P.R.: Design of a device with wavelength multiplexing and ronting functions using dynamic holography on SLMs. Opt. Commun. J. 263(2), 166-170 (2006)

Parker. M.C. Cohen. A.D. Mears, R.J.: Programmable Holographic Elements for WDM. IEE-Colloquiunon-Opteelectronic-Integration-and-S witching-Ref.-No.1997/372. 1997: 10/1-6 IEE. (1997)

Parker, M.C., et al.: Dynamic digyital holographic wavelength filtering. J. Lightwave. Tech. 16(7), 1259$1270(1998)$

Riza, A., Yuan, S.: Reconfigurable wavelength Add-Drop filtering based on a Banyan network topology and Ferroelectric Liquid Crystal tiber optics switches. J. of Lightwave Technology 17(9), 1575-1584 (1999)

Sadot, D. Boimovich. E.: Tunable optical filters for dense WDM networks. IEEE Communications Magazine. pp. 50-55, Dec (1998)

Tebben. D., Nolan. D. et al.: Two-Fiber Optical Shared Protection Ring with Bi-ditectional 4 \%. 4 Optical Switch Fabrics LEOS 2001. The 14th Antual Meeting of the IEEE, vol. 1, pp. 228-229 (2001) 